EPJ Web of Conferences 92,02038 (2015)

DOI: $10.1051 /$ epjconf/20159202038

(C) Owned by the authors, published by EDP Sciences, 2015

\title{
Experimental and numerical investigation of an air to air supersonic ejector for propulsion of a small supersonic wind tunnel
}

\author{
Jan Kracík ${ }^{1, a}$, Václav Dvořák ${ }^{1}$ \\ ${ }^{1}$ Department of Power Engineering Equipment, Faculty of Mechanical Engineering, Technical University of Liberec, \\ Studentská 2, 46117, Liberec, Czech Republic
}

\begin{abstract}
The article deals with experimental and numerical investigation of an air to air supersonic ejector with twelve primary nozzles. The ejector is supposed to be used for propulsion of a small experimental supersonic wind tunnel which is situated in laboratories of Technical University of Liberec. A novel arrangement with 12 primary nozzles is used. The nozzles are placed at the periphery of the mixing chamber. The secondary stream enters the ejector through the free centre of the mixing chamber and is sucked into the space between the primary nozzles. Moreover the declination of the primary nozzles towards to ejector axis is $8.2^{\circ}$ and the shape of the mixing chamber and diffuser walls is given by normal cubic spline function, which was investigated in previous work. The declination of the primary nozzles is supposed to eliminate reversal flow in the centre of the mixing chamber. Experimental results for different numbers of simultaneously activated primary nozzles are carried out. Experimental results are compared to the numerical simulation made with the help of Ansys Fluent software.
\end{abstract}

\section{Introduction}

Recently there are still higher requirements for working efficiency of engineering devices and optimal working regimes are required. The ejectors are not an exception, on the contrary, these devices, with their generally low efficiency (less than $30 \%$ ), might need more attention than other machinery. The low efficiency is probably the greatest disadvantage of these devices. On the other hand, advantages of the ejectors are especially absence of moving parts, ointment or seals. Other advantages are a simply design and a relatively cheap manufacturability. They are mostly used in plants where sufficient amount of the working fluid is available.

By the time that Keenan at al [1] performed the first comprehensive study of mixing, two cases of mixing were distinguished: the constant pressure mixing and the constant area mixing. From this follows usage of the ejectors. Another division of the ejectors is in terms of primary and secondary streams arrangement. The primary stream can either be supplied in the ejector axis and secondary stream is sucked at the periphery of the mixing chamber or primary stream enters the periphery of the mixing chamber and the secondary stream is sucked in the ejector axis, which is especially used for wind tunnel propulsions. In addition the second case can

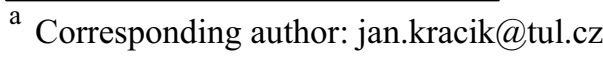

be designed in two arrangements. The first arrangement uses a slot primary nozzle. The other arrangement uses several primary nozzles, see figure 1.

The design of the air to air ejector in this work was investigated in details in work [2]. The ejector is used for propulsion of a small experimental variable wind tunnel [4] and its primary stream is supersonic, which makes the investigation more complex. As can be seen in figure 1 , the ejector consists of twelve primary supersonic nozzles which are placed at the periphery of the mixing chamber and their declination towards the ejector axis is $8.2^{\circ}$. The declination should prevent reversal flow in the ejector axis, as was shown in work [3].

\section{Methods}

The aim of the work is to investigate the supersonic ejector with different count of simultaneously activated primary nozzles. For the first experiments, three cases of simultaneously activated nozzles were chosen: the four, eight and all twelve primary nozzles. Compressed air supplied from pressure tanks was the primary medium. The secondary medium - atmospheric air was sucked in direction of the ejector axis. The measurements of the static pressure on the mixing chamber wall have been done. Stagnation pressure of the primary air was $\mathrm{p}_{01}=400 \mathrm{kPa}$ (absolute) and stagnation temperature was $\mathrm{T}_{01}=295 \mathrm{~K}$. Stagnation 
pressure of the secondary air was $\mathrm{p}_{02}=96 \mathrm{kPa}$ (absolute) and stagnation temperature was considered the same as in the primary air, i.e. $\mathrm{T}_{02}=\mathrm{T}_{01}$. All measurements were provided for zero backpressure $\left(\mathrm{p}_{\mathrm{b}}\right.$ $=\mathrm{p}_{02}=96 \mathrm{kPa}$ ). Arrangement during the experiment is obvious from figure 2 .

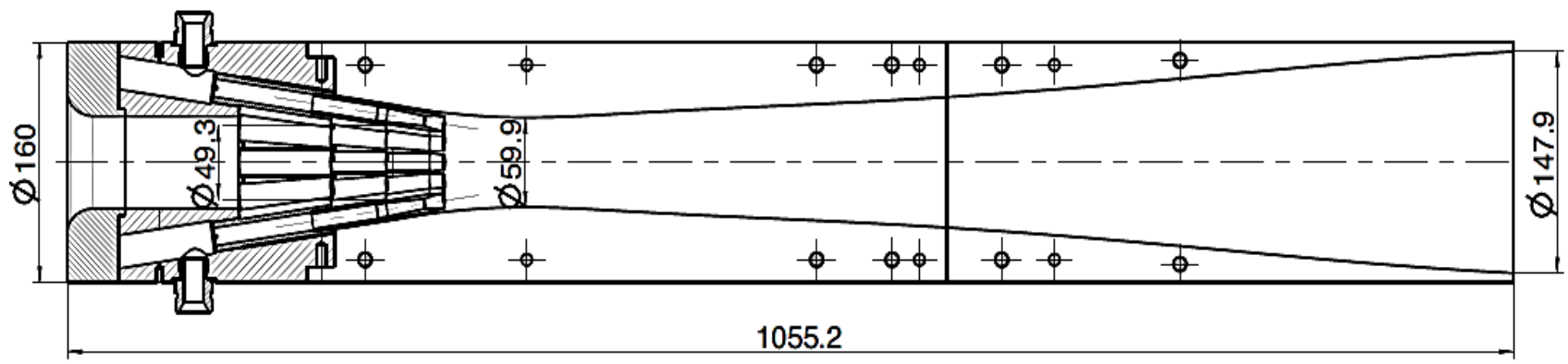

Figure 1. Longitudinal cross-section through the ejector axis with main dimensions in millimetres.

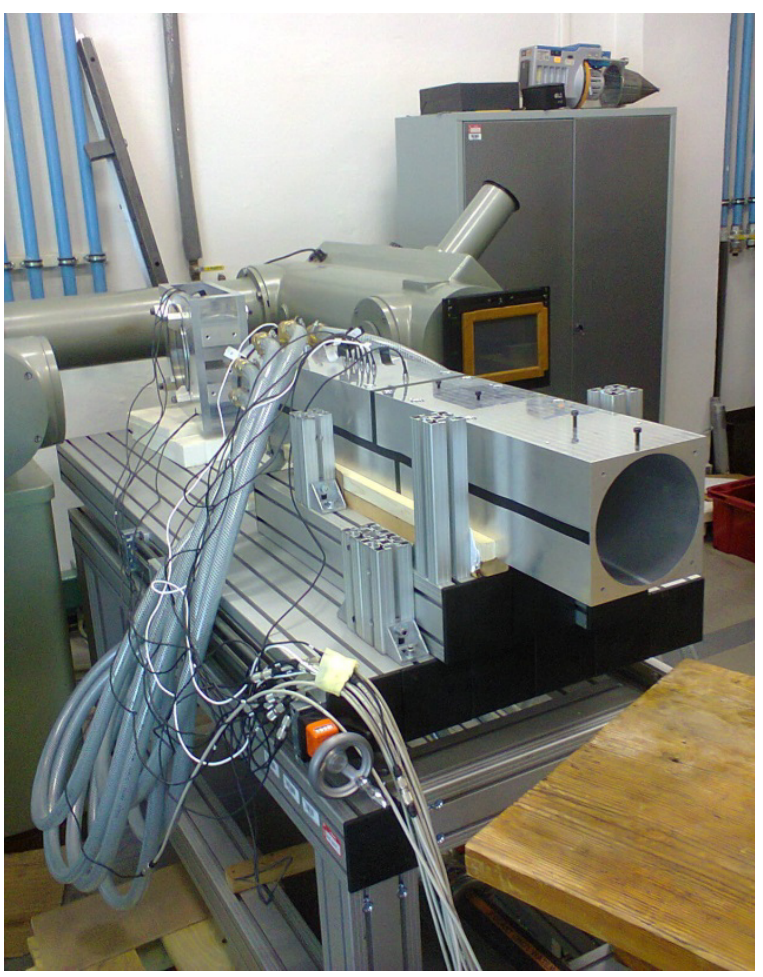

Figure 2. The supersonic ejector during the experiment.

Twelve piezoelectric pressure sensors were used for acquisition of static pressure distributions on the mixing chamber wall. Others two sensors were used for measurement of stagnation pressure of the primary and the secondary flow. Adjustable valves for each of the primary nozzle are shown in figure 3.

Numerical simulation has been done only for arrangement with all twelve simultaneously activated primary nozzles. Because of the ejector design an axisymmetric calculation was not possible. Therefore, a computational model had to be solved as a threedimensional but symmetrical along two planes that form an angle of $30^{\circ}$. Thus, the final model was created as a twelfth of the inner channel of the experimental device and contained the whole one primary nozzle. Boundary conditions were set in the same way as in the experiment. There was a density based solver used due to supersonic character of the flow unlike the previous work [3] where a pressure based solver was used for a better convergence. An implicit formulation and a second order upwind scheme were used. The total cross-section area of the throats of the primary nozzles is $396 \mathrm{~mm}^{2}$.

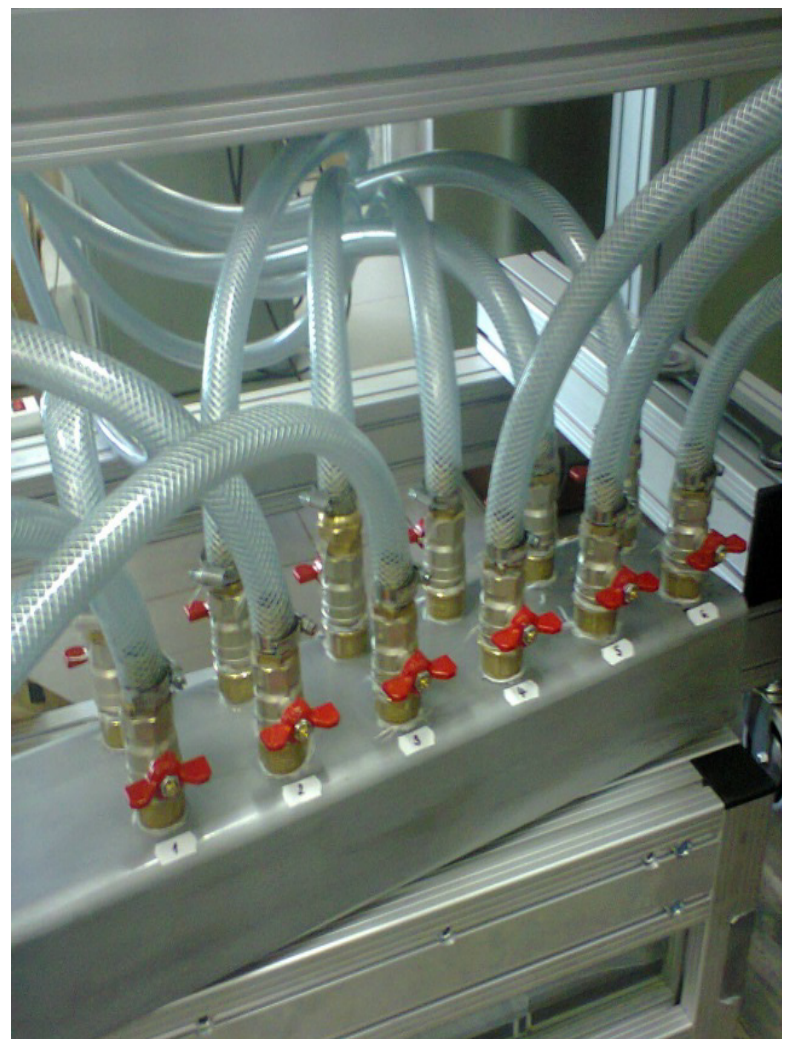

Figure 3. Intake of the primary air into the nozzles with adjustable valves. 


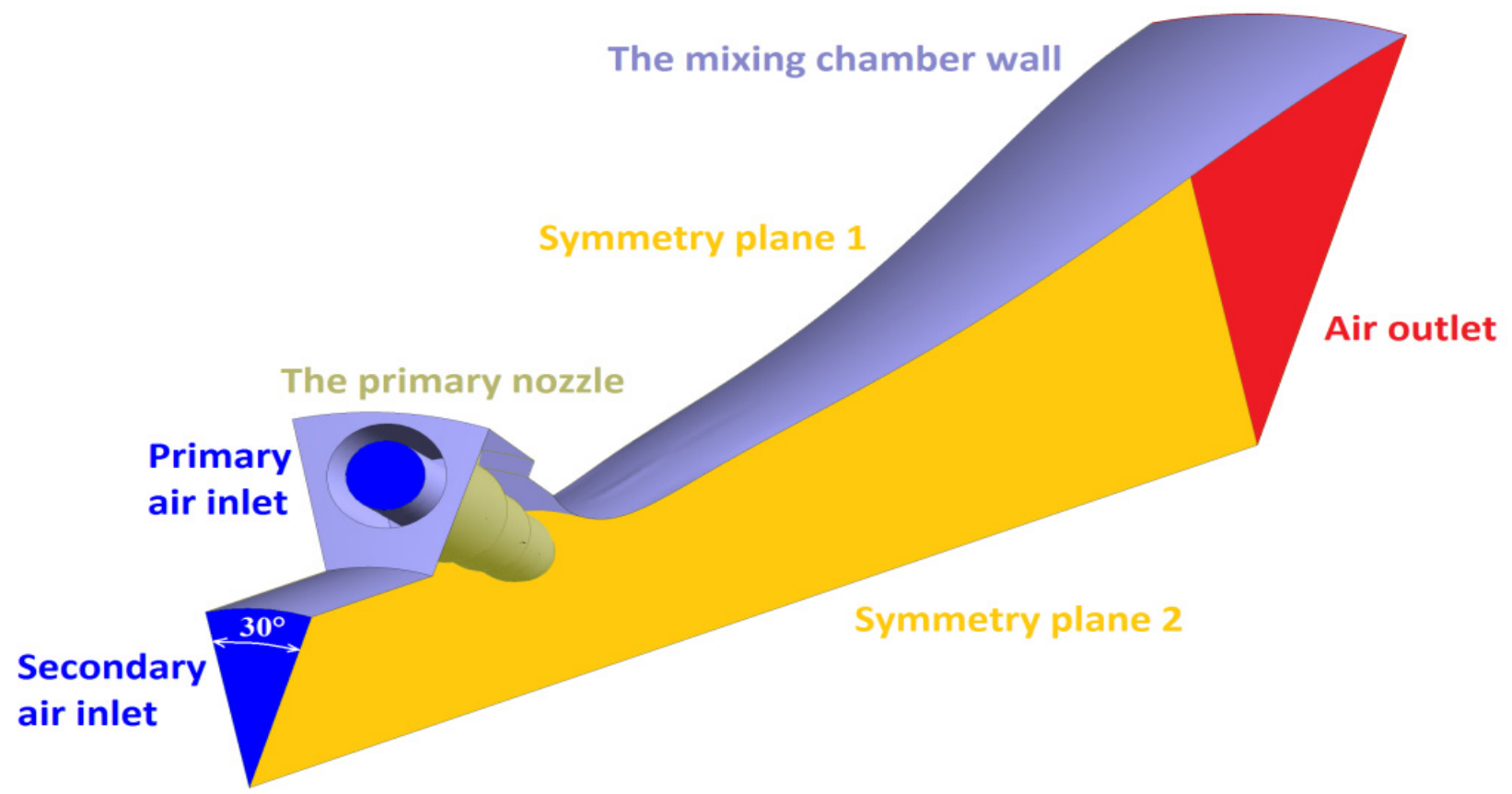

Figure 4. Computational model of the ejector with boundary conditions.

Each of the primary nozzles is designed for exit Mach number $M_{n}=2.2$. The cross-section area of the secondary air inlet is approximately $2980 \mathrm{~mm}^{2}$.

$\mathrm{K}-\omega$ SST turbulence model was used in this case. This model is suitable for calculation of flow in supersonic ejectors as it was stated in previous works $[5,6,7]$. For further information see documentation [9].

\section{Results}

\subsection{Experiment}

Experimental arrangements for investigated cases are shown in figure 5. Comparison of static pressure distributions on the mixing chamber wall obtained for all three cases are carried out in figure 6 . These results were obtained for absolute stagnation pressure of primary air $\mathrm{p}_{01}=400 \mathrm{kPa}$. For 4 activated primary nozzles, the lowest reached value of the static pressure is in the region in front of the ejector throat - the narrowest cross-section of the mixing chamber - and is about $75 \mathrm{kPa}$ (absolute). Slow increase of the static pressure can be seen right behind the ejector throat. The value of the static pressure indicates subsonic flow in the mixing chamber. In the second case for 8 activated primary nozzles, the lowest value of the static pressure is, on the contrary of the previous case, reached behind the ejector throat and this value is about $44 \mathrm{kPa}$ (absolute). Due to proceeding expansion behind the throat, we can assume that the flow is supersonic in this region. The expansion took place up to the distance 20 $\mathrm{mm}$ behind the ejector throat and then relatively rapid increase of the static pressure occurred. The expansion behind the throat is also present for the last case with all
12 simultaneously activated primary nozzles. Here, the decrease of the static pressure is up to the distance of $180 \mathrm{~mm}$ behind the throat. The value is about $23 \mathrm{kPa}$ (absolute) in this region. The static pressure rising is even more rapid compare to the previous case with 8 activated primary nozzles. The sudden growths of static pressure for cases of 8 and 12 activated primary nozzles are probably caused by a normal shock wave behind the ejector throat, which can be the reason of high pressure losses in the mixing chamber.
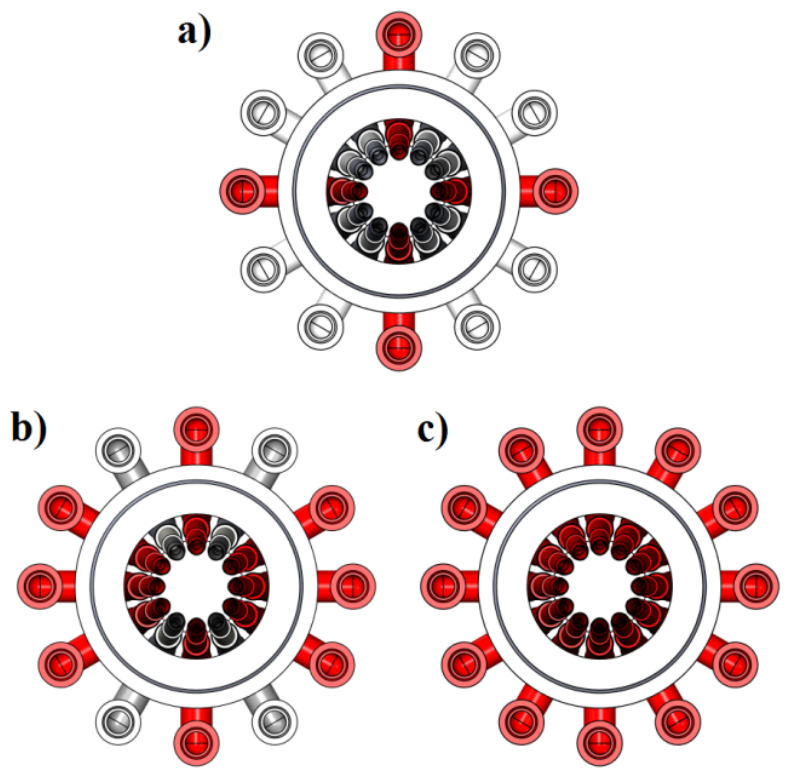

Figure 5. Experimental arrangement for 4(a), 8(b) and 12(c) primary nozzles. 


\subsection{Numerical simulation}

The results of the numerical simulation are showed in figure 7. There are contours of Mach number and static pressure in the figure. Both the Mach number and the static pressure contours show the existence of the normal shock wave as it has been indicated from the experimental data. No reversal flow can be observed in the mixing chamber. In earlier works [2, 3], the existence of the normal shock wave was not proved, because lower stagnation pressure on the boundary condition of the secondary air inlet was set. The intention was a presumption of a normal shock wave in the test section of the supersonic wind tunnel. Comparison of the numerical simulation and the experimental data for 12 simultaneously activated primary nozzles shows figure 8 . A very good agreement was achieved. Calculated static pressure course behind the ejector throat is slightly higher than measured, but the position of the normal shock wave is predicted very precisely.
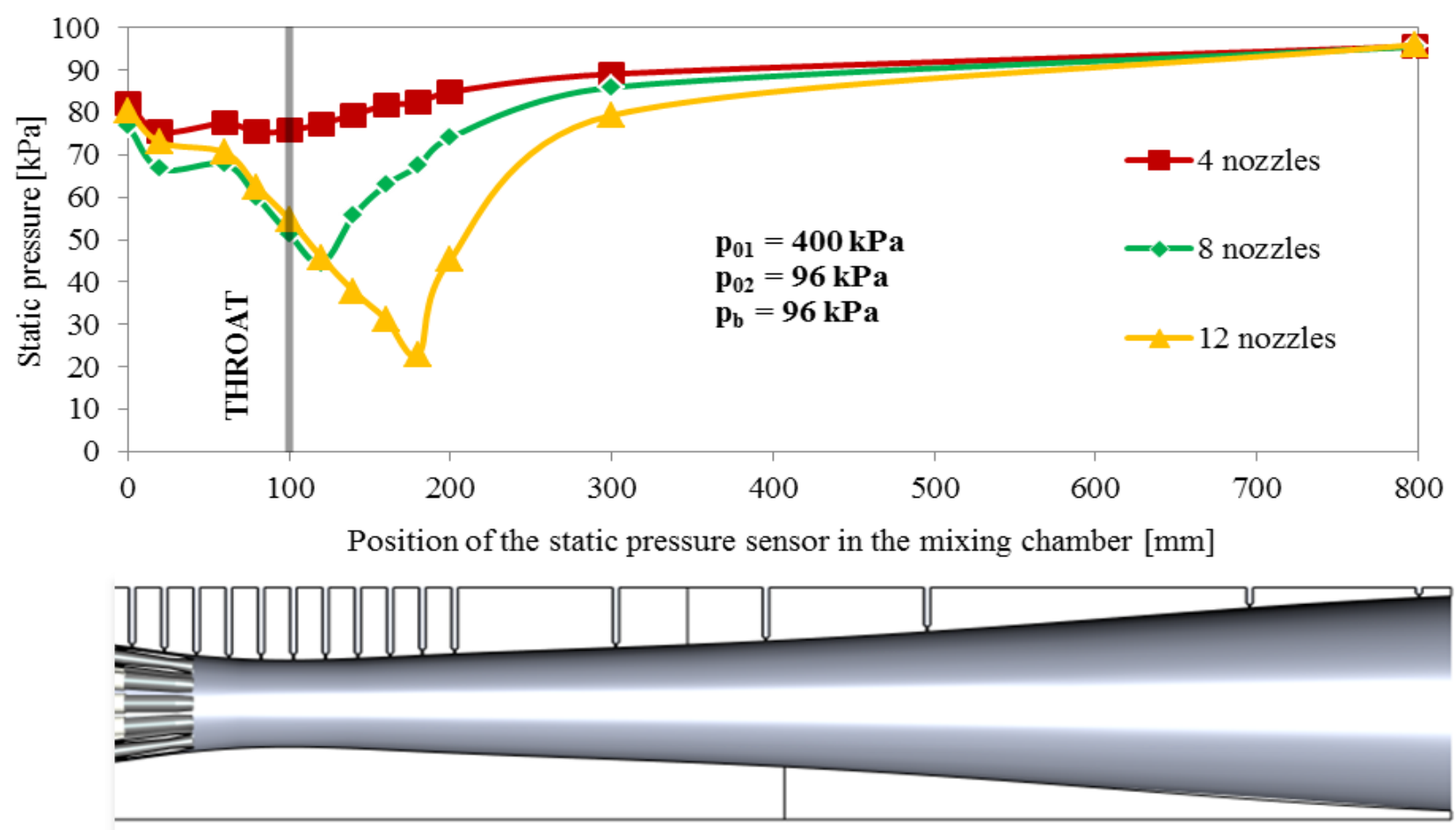

Figure 6. Experimental data of the static pressure distribution on the mixing chamber wall for 4, 8 and all 12 simultaneously activated primary nozzles.

a)

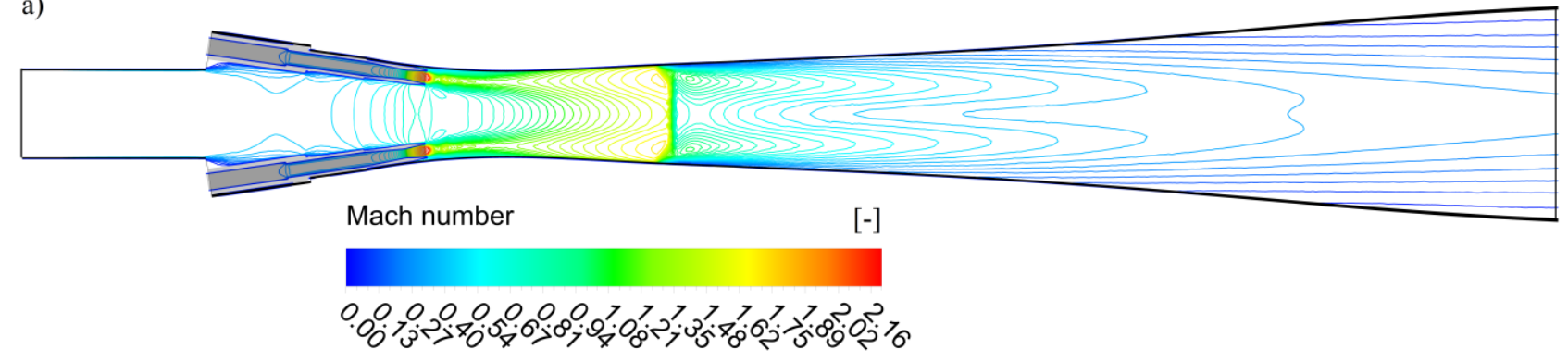

b)

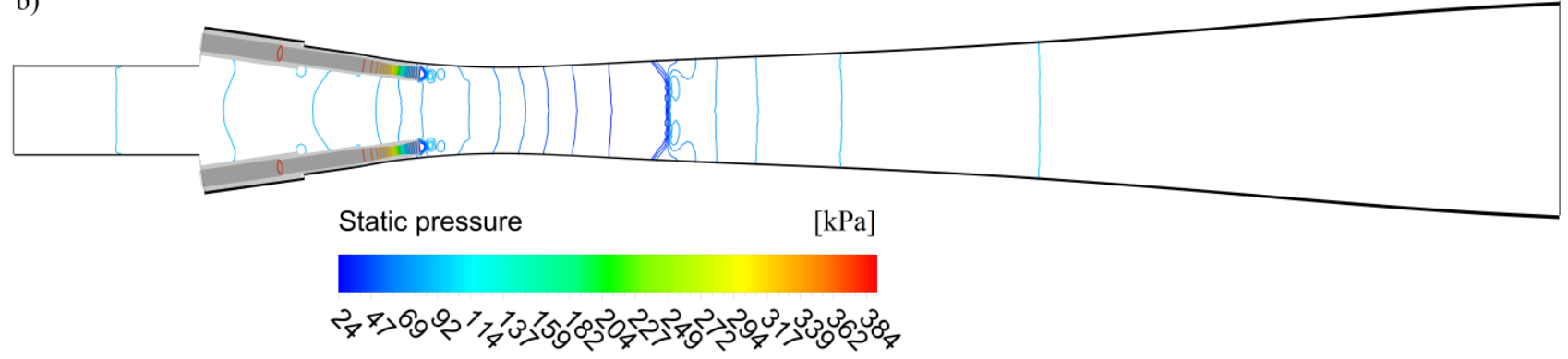

Figure 7. Contours of Mach number (a) and static pressure (b) for all 12 simultaneously activated primary nozzles. 


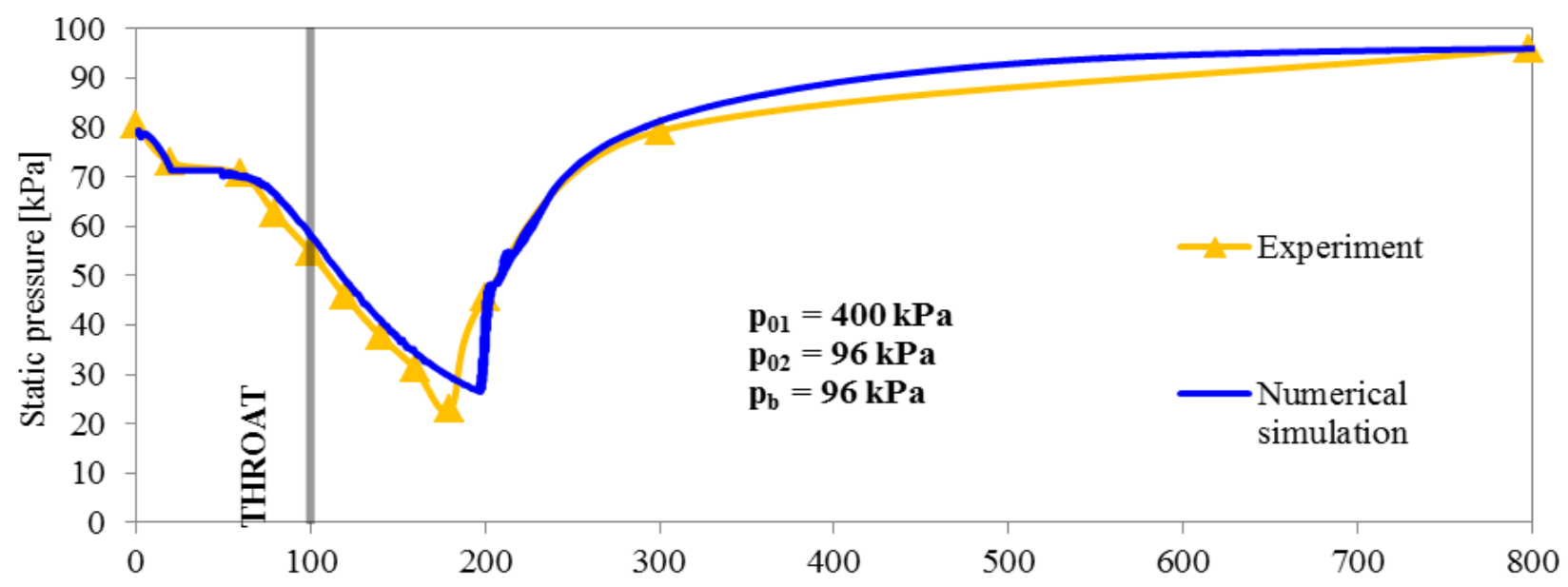

Position of the static pressure sensor in the mixing chamber $[\mathrm{mm}]$

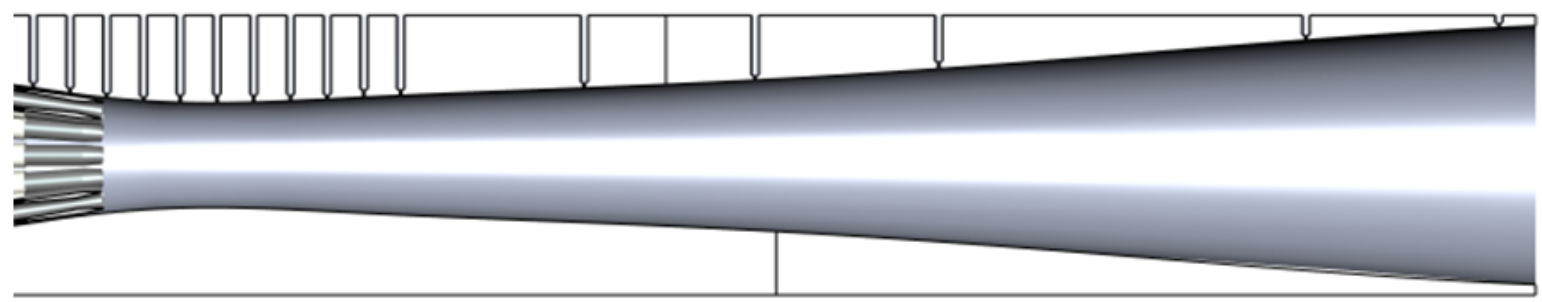

Figure 8. Comparison of the experimental and numerical data for all 12 simultaneously activated primary nozzles.

\section{Conclusions}

The first experimental results for three cases simultaneously activated primary nozzle have been presented. The supersonic ejector was investigated without a connection to a supersonic wind tunnel. The case with 4 activated primary nozzles is not interesting enough due to not reaching supersonic velocities in the mixing chamber. This case is not suitable for propulsion of a supersonic wind tunnel due to low sucking power of the ejector. On the contrary, supersonic velocities behind the ejector throat are reached for the cases with 8 and 12 activated primary nozzles. Existence of a normal shock wave in the mixing chamber behind the ejector throat was proved. Further, a comparison of the experimental and numerical data for 12 simultaneously activated primary nozzles has been done. There was a relatively good agreement achieved.

\section{Acknowledgment}

This project was realized with financial support by the Czech Science Foundation, grant no. P101/10/1709 and by the University Development Fund of the Czech Ministry of Education, Youth and Sports, grant no. 573/2013/G1.

\section{References}

1. J. H. Keenan, E.P. Neumann and F. Lustwerk, Journal of Applied Mechanics, Trans ASME, 72, pp A75 - A81 (1950).

2. J. Kracik, V. Dvorak, J. Kolar, Eur. Phys. J. E 67, 4 (2014)

3. V. Dvorak, Shape Optimization of Supersonic Ejector for Supersonic Wind Tunnel.(Applied and Computational Mechanics, Plzen, 2010)

4. J. Kracik, Design of Supersonic Wind Tunnel (Diploma thesis, In Czech, 2014)

5. J. Kolar, V. Dvorak, Interaction of Shock Waves in Supersonic Ejector (Proceedings of the 27th Meeting of Thermodynamics and Fluid Mechanics Departments, Plzen, 2008)

6. J. Simak, Computation of the Flow and Interaction of Shock Waves in a 2D Supersonic Ejector (Colloquium Fluid Dynamics 2009, Prague, 2009)

7. Y. Bartosiewicz, Z. Aidoun, P. Desevaux, Y. Mercadier, Numerical and Experimental Investigations on Supersonic Ejectors (International Journal of Heat and Fluid Flow 26, 2005)

8. J. Kolar, Design and Optimization of Ejector for Supersonic Wind Tunnel (Diploma thesis, Liberec, In Czech, 2007)

9. Ansys Fluent 15.0 User's Guide, Ansys Inc. 